\title{
Antibiogram of single, double and triple chain Aroyl hydrazine against some gram positive and gram negative bacteria.
}

\author{
Parvin $\mathrm{R}^{1}$, Absar $\mathrm{MN}^{2}$, Ershaduzzaman $\mathrm{M}^{3}$, Mahbub-E-Elahi $\mathrm{ATM}^{4} *$, Shil A \\ ${ }^{1}$ Sylhet Govt. College, ${ }^{2}$ Department of Chemistry, Jahangirnagar University, ${ }^{3}$ Bangladesh Livestock Research \\ Institute, Savar, ${ }^{4}$ Department of Microbiology\& Hygiene, Sylhet Agricultural University, ${ }^{5}$ Department of \\ Botany, Jahangirnagar University, Bangladesh.
}

[Received: 12 January 2011, Accepted: 16 March 2011]

\begin{abstract}
The antibacterial sensitivity of some single, double and triple chain aroylhydrazine against gram positive and gram negative bacteria were performed by disc diffusion method. Most of the compounds showed appreciable antibacterial activity against different gram positive and gram negative bacteria. The single chain hydrazines are more active then double chain and triple chain hydrazine. Among the single chain aroylhydrazines studied only 4-n-hexyloxy benzoyl hydrazine is the most active. The significant activity of 4-n-hexyloxybenzoyl hydrazine and heptyloxybenzoyl hydrazine against gram positive and gram negative bacteria may be (formation of inhibition zone 8 to 22 mm with most of the test bacteria) due to their liophilicity of the bacterial cell membrane. Anti-microbial activity decreases as the number of carbon of single chain hydrazine increases $\left(\mathrm{C}_{6}>\mathrm{C}_{7}>\mathrm{C}_{8}>\mathrm{C}_{9}>\mathrm{C}_{10}\right.$ single chain hydrazine). Double chain hydrazines $(3,5$ or 3,4$)$ are more active than triple chain hydrazines $(3,5>3,4>3,4,5$ hydrazine). The antibacterial activities of hydrazines are being decreased as their increasing number of side chain.
\end{abstract}

Keyword: Antibacterial sensitivity, Aroyl hydrazine

\section{INTRODUCTION}

Antitumour properties of some amino acid complexes of copper (II) have already been investigated ${ }^{[1]}$. Most of the heterocyclic amines are used as corrosion inhibitors and their complexes with platinum and copper have been tested as antitumour ${ }^{[2]}$ and antibacterial ${ }^{[3]}$ agents and 3-amino pyridine has strong anticonvulsive effects [4, 5]. Some coordination complexes have already been appeared in the literature ${ }^{[6,7]}$ as antibacterial agents. For investigating liquid crystal properties of copper complexes we have been synthesized copper complexes, aroylhydrazines, aroylhydrazones. Biological activities of $\mathrm{N}$-salicylideneacylhydrazines have already been investigated. If 3-aminopyradine has strong anticonvulsive effects and $\mathrm{N}$ salicylideneacylhydrazines show a wide spectrum of biological activities. Aroyl hydrazines have free amino group. So hydrazines may have antibacterial activity. The above information motivates us to study the antibacterial activity of aroyl hydrazines. Therefore in the present paper report the study of the antibacterial activity of single chain, double chain and triple chain aroyl hydrazine. Pure culture strain of ten gram positive and gram negative bacteria were used in this study.

\section{MATERIALS AND METHODS}

The susceptibility of the microorganisms to antimicrobial agents may be measured in vitro by utilizing agar diffusion technique provided that all the procedural details are carefully controlled. Dried filter paper discs containing the test materials are usually applied to the test plates containing the culture of microorganisms. The dried discs adsorb water from the agar medium and the test material is dissolved. Then the test material diffuses through the adjacent agar medium according to the physical law that governs diffusion of molecules through an agar gel. As a result there is a gradual change of test material concentration in the agar surrounding each disc. Activities of the test sample are expressed by measuring the zone of inhibition observed around the area. The zone of inhibition is affected by various factors, by the growth rate of the microorganism, rate of diffusion of test material through the agar gel and concentration of test materials. The diameter of the inhibition zone is usually measured in culture media to understand the extent of inhibition in different concentration. Used gram positive bacteria are Bacillus cereus, Bacillus megaterium, Bacillus subtilis, Staphylococcus aureus and the gram negative bacteria Shigella sonnei, Shigella dyscenteriae, Shigella Flexner, Salmonella typhae, Salmonella paratyphae A, Escherichia coli. Individual bacterial strains (gram positive and gram negative) in pure state were obtained from Department of Food and Nutrition, University of Dhaka. There are two common culture media used for the cultivation of bacteria. They are Nutrient broth (HIMEDIA) M002, Nutrient agar (HIMEDIA) M001.

\section{Preparation of wet disc for sensitivity test}

The anti-microbial sensitivity test was performed by disc diffusion method ${ }^{[8]}$. Punched discs of $8 \mathrm{~mm}$ in diameter from number 1 whatman filter paper and 
dispersed batches of 100 discs in screw capped bottles were sterilized in autoclave. In an aseptic condition the test material hydrazine was allowed to absorb into the filter paper disc $(400 \mu \mathrm{gm} /$ disk $)$ and left over night for complete removal of solvent (dichloromethane).

\section{Anti-microbial assay procedure}

The test organisms from the pure culture were transferred to the slant with the help of an inoculation loop in an aseptic condition. After inoculation the slant was subjected to incubation at $37^{\circ} \mathrm{C}$ for $12-24$ hours to provide sufficient time and temperature for the growth of the test organisms. Cultures were used within two or three days. The test organisms from slant were transferred to nutrient broth and incubate at $37^{\circ} \mathrm{C}$ for 24 hours for sufficient growth. After incubation turbidity was observed in the test tube, it was ready to use as test organisms. On the other hand nutrient agar was poured into Petri dishes on a level horizontal surface so as to give a uniform depth of approximately $4 \mathrm{~mm}$. After the medium had been allowed to cool to room temperature it was stored in a refrigerator.

Just before use the plates were placed in an incubator $\left(25^{\circ} \mathrm{C}\right)$ with lids for about $10-15$ minutes, until excess surface moisture was lost by evaporation. There should be no droplets of moisture on the surface of the medium or on Petri dish plate cover. The test organism was immediately transferred to the Petri dish culture media (nutrient agar) in aseptic condition in order to facilitate a homogenous distribution of test organisms the Petri dish was rotated several times first clockwise and then anti clockwise. The discs were placed on the plate with a sterile forceps. The plates were then kept in a refrigerator for about 4 hours in order to allow sufficient time to the test material to diffuse to considerable areas of the plate. The plates were then incubated at $37^{\circ} \mathrm{C}$ for $36-72$ hours in an incubator. The anti-microbial activity was detected by the formation of a clear zone around the disc. The zone of inhibition was measured in $\mathrm{mm}$ from the under surface of the Petri dishes using a ruler ${ }^{[9]}$.

\section{Synthesis of hydrazine}

All chemicals were obtained from ACORS chemicals and were used without further purification. Except ethanol and 1-butanol distilled over anhydrous calcium oxide and dichloromethane was distilled over anhydrous $\mathrm{P}_{2} \mathrm{O}_{5}$. Infrared spectra in the range $4000-400 \mathrm{~cm}^{-1}$ were recorded from an intimated mixture of the compounds and $\mathrm{KBr}$ using a JASCO FT-IR-460+TLUS spectrometer. The ${ }^{1} \mathrm{H}-\mathrm{NMR}$ spectra were recorded either on Jeol MY60 FT NMR (to $\mathrm{MHz}$ ) machine or on Bruker DPX 400 spectrometer $(400 \mathrm{MHz})$ Pre-coated silica gel glass plate (Silica gel 60, F-254, $0.25 \mathrm{~mm}$ ) from E-Merck was used for analytical TLC while for separative purpose flash column chromatography was done over silica gel Merck 60 (230-400 mesh).
Synthesis of alkoxybenzoates: (1a-23a Scheme-1) All the single, double and triple chain alkyloxybenzoates were synthesized by using a general procedure, the synthetic routes shown in scheme-1, a representative detail for ethyl-4-nhexyloxybenzoate is given below:

Ethyl-4-hydroxybenzoate (1 mole) and 1 bromoalkane $(1 \times 1.2$ mole ratio) mix together with anhydrous potassium carbonate $(1 \times 1.5$ mole ratio), made a salary and was irradiated with microwave radiation at medium law power $(384 \mathrm{w})$ for 3.5 minutes (Scheme-1). Extraction of the reaction product with dichloromethane and hexane $(2: 1 \mathrm{v} / \mathrm{v})$ followed by chromatographic purification over silica gel gave ethyl-4-n-hexyloxy-benzoate.

\section{1a. Ethyl-4-n-hexyloxybenzoate:}

Yield: $85 \%$.

IR (liquid film): $v, \quad \mathrm{~cm}^{-1}: \quad 2925(\mathrm{~s}), \quad 2855(\mathrm{~m})$ (aliphatic C-H), 1727(s) (C=O), 1608(m), 1541(w), 1496(w) (aromatic C=C), 1475(m), 1468(w), 1365(m), 1215(m), 1111(m), 1034(w), 924(w), 865(w), 766(w), 725(w).

$\left.{ }^{1} \mathbf{H}-\mathbf{N M R}\left(\mathbf{C D C l}_{3}\right) \mathbf{( 2 5 0} \mathbf{M H z}\right) \delta, \mathrm{ppm}: 7.89(2 \mathrm{H}, \mathrm{d}$ $\mathrm{J}=9 \mathrm{~Hz})$ and $6.88(2 \mathrm{H}, \mathrm{d} \mathrm{J}=9 \mathrm{~Hz}), 4.35(2 \mathrm{H}, \mathrm{q}$, $\left.\mathrm{J}=7.0 \mathrm{~Hz},-\mathrm{COOC}_{2} \mathrm{CH}_{3}\right), 4.01(2 \mathrm{H}, \mathrm{t}, \mathrm{J}=6.5 \mathrm{~Hz},-$ $\left.\mathrm{OCH}_{2} \mathrm{CH}_{2}-\right), 1.79\left(2 \mathrm{H}, \mathrm{m},-\mathrm{OCH}_{2} \underline{\mathrm{C}}_{2} \mathrm{CH}_{2}-\right), 1.38$ $\left(3 \mathrm{H}, \mathrm{t}, \mathrm{J}=7.0 \mathrm{~Hz},-\mathrm{COOCH}_{2} \mathrm{CH}_{3}\right), 1.2-1.5[8 \mathrm{H}, \mathrm{m},-$ $\left.\mathrm{OCH}_{2}\left(\mathrm{CH}_{2}\right)_{4} \mathrm{CH}_{3}\right], \quad 0.88 \quad[3 \mathrm{H}, \quad \mathrm{J}=6.5 \mathrm{~Hz}, \quad-$ $\left.\mathrm{O}\left(\mathrm{CH}_{2}\right)_{5} \underline{\mathrm{C}}_{3}\right]$.

Synthesis of Alkyloxy benzoyl hydrazine (1b-23b Scheme-2-5):

All the single, double and triple chain alkyloxy benzoyl hydrazines were synthesized by using a general procedure; the synthetic routes have shown in scheme-2-5, a representative detail for 4-nhexyloxy benzoyl hydrazine is given below:

A mixture of ethyl-4-n-hexyloxybenzoate and hydrazine hydrate, in 1:10 molar ratio, was refluxed in 1-butanol $(25 \mathrm{~mL})$ for 36 hours. The reaction mixture was cooled to room temperature and treated with water $(\approx 250 \mathrm{~mL})$. The white solid thus formed was filtered and dried under suction, and recrystallised from hot ethanol to give the pure required compounds (Scheme-2,1b).

\section{1b. 4-n-hexyloxy benzoyl hydrazine:}

Yield: $78 \%$.

IR (KBr): v, $\mathrm{cm}^{-1}:$ 3322(w), 3158(w) (N-H), 2919(w), 2856(w) (aliphatic C-H), 1640(m) $(\mathrm{C}=\mathrm{O})$, 1619(m) (N-H, bending) 1578(w), 1510(w),1501(m) (aromatic C=C), 1468(w), 1426(m), 1347(m), 1235(m), 1227(s), 1071(w), 922(w), 853(w), 768(w), 725(w), 658(w).

${ }^{1}$ H-NMR $\left(\mathbf{C D C l}_{3}\right)(\mathbf{6 0 M H z}) \delta$, ppm: $\delta 7.75(2 \mathrm{H}, \mathrm{d}$, $\left.\mathrm{J}=9 \mathrm{H}_{2}\right)$ and $6.9\left(2 \mathrm{H}, \mathrm{d}, \mathrm{J}=9 \mathrm{H}_{2}\right) 7.27(1 \mathrm{H}, \mathrm{s}$, $\left.\mathrm{CON}_{\mathrm{HNH}}\right), 4.06\left(2 \mathrm{H}, \mathrm{s},-\mathrm{CONHN} \underline{\mathrm{H}}_{2}\right), \delta 4.01(6 \mathrm{H}, \mathrm{t}$, $\left.\mathrm{J}=5.5 \overline{\mathrm{Hz}}, \quad-\mathrm{OCH}_{2} \mathrm{CH}_{2}-\right), \quad \delta 1.91-\quad 1.32 \quad[8 \mathrm{H}, \mathrm{m}, \quad-$ 
$\left.\mathrm{OCH}_{2}\left(\mathrm{CH}_{2}\right)_{4} \mathrm{CH}_{3}\right]$ and $\delta 0.90$ [3H, overlapped triplet, $\left.-\mathrm{O}\left(\mathrm{CH}_{2}\right)_{3} \mathrm{CH}_{2} \mathrm{CH}_{3}\right]$.

The synthetic routes of Alkoxy esters and Alkyloxy benzoyl hydrazines are shown as follows:

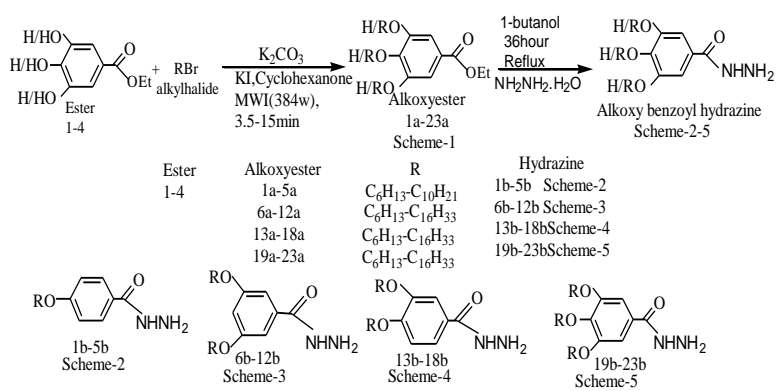

\section{RESULTS}

The Alkoxy esters (1a-23a) were synthesized by reacting with hydroxy ester (1-4) and alkyl halides in presence of $\mathrm{K}_{2} \mathrm{CO}_{3}$, KI (Scheme-1), 4-Alkoxy ester, 3,5-Alkoxy ester, 3,4-Alkoxy ester, 3,4,5-Alkoxy ester react with hydrazine hydrate to form corresponding aroylhydrazine (1)-23b,Scheme-2-5). All the compounds were characterized by IR and ${ }^{1} \mathrm{H}-$ NMR spectra.

Table-1: Anti-bacterial activity of single chain hydrazine: $\left(\mathrm{R}-\mathrm{C}_{6} \mathrm{H}_{13}-\mathrm{C}_{10} \mathrm{H}_{21}\right.$, Scheme-2)

\begin{tabular}{|c|c|c|c|c|c|c|}
\hline \multirow[t]{2}{*}{ Test Organism } & \multirow{2}{*}{$\begin{array}{l}\text { Con of test } \\
\text { material } \\
\mu \mathrm{g} \text { disk }\end{array}$} & \multicolumn{2}{|c|}{$\begin{array}{l}\text { Zone of in } \\
\text { compounds }\end{array}$} & \multirow{2}{*}{$\begin{array}{l}\text { (mm) } \\
3 \mathrm{~b} \\
\mathrm{C}_{8} \mathrm{H}_{17}\end{array}$} & \multirow{2}{*}{$\begin{array}{l}\text { of actic } \\
4 \mathrm{~b} \\
\mathrm{C}_{9} \mathrm{H}_{19}\end{array}$} & \multirow{2}{*}{$\begin{array}{l}\text { of the } \\
5 \mathrm{~b} \\
\mathrm{C}_{10} \mathrm{H} \\
21\end{array}$} \\
\hline & & $\begin{array}{l}1 \mathrm{~b} \\
\mathrm{C}_{6} \mathrm{H}_{1} \\
3\end{array}$ & $\begin{array}{l}2 \mathrm{~b} \\
\mathrm{C}_{7} \\
\mathrm{H}_{15}\end{array}$ & & & \\
\hline Shigella sonnei. & 400 & 8 & 8 & 15 & - & 10 \\
\hline Bacillus cereus. & 400 & $22^{*}$ & 12 & 15 & - & 8 \\
\hline Salmonella typhae & 400 & 19 & 6 & - & - & 16 \\
\hline Shigella dyscenteriae. & 400 & 15 & 8 & 8 & - & - \\
\hline Salmonella paratyphae A & 400 & 10 & - & - & - & - \\
\hline Bacillus subtilis. & 400 & 12 & 15 & 12 & - & - \\
\hline Staphylococcus aureus & 400 & 16 & 12 & 8 & 5 & - \\
\hline Escherichia coli & 400 & 10 & 10 & - & 8 & 8 \\
\hline Shigella flexneri & 400 & 12 & - & 10 & - & 5 \\
\hline Bacillus megaterium & 400 & 12 & 14 & 10 & 6 & 8 \\
\hline
\end{tabular}

Zone size more than $13 \mathrm{~mm}$ highly sensitive, Zone size $8-12 \mathrm{~mm}$ moderately sensitive Zone size $1-7 \mathrm{~mm}$ less sensitive, $\quad--=$ no inhibition spectrum, $\quad *$ h highest inhibition spectrum, $\square$ means 12 hours, $1 b=4$-n-hexyloxy benzoyl hydrazine, $2 b=4$-n-heptyloxy benzoyl hydrazine, $3 \mathrm{~b}=4$-n-octyloxy benzoyl hydrazine, $4 \mathrm{~b}=4$-n-nonyloxy benzoyl hydrazine, $5 \mathrm{~b}=4$-n-decyloxy benzoyl hydrazine.

Antibacterial activities of different aroylhydrazines (test materials) were measured by the zone inhibition technique expressed by average diameter. These activities were measured against different microorganisms and the results summarized as follows (Table-1, 2, 3, 4) and antibacterial sensitivity of some antibiotics given table- 5 .
Table-2: Anti-bacterial activity of $(3,5)$ substituted hydrazine: $\left(\mathrm{R}-\mathrm{C}_{6} \mathrm{H}_{13}-\mathrm{C}_{16} \mathrm{H}_{33}\right.$, Scheme-3)

\begin{tabular}{|c|c|c|c|c|c|c|c|}
\hline \multirow{2}{*}{ Test Organism } & \multirow{2}{*}{$\begin{array}{l}\text { Con of } \\
\text { test } \\
\text { material } \\
\mu \mathrm{g} \text { disk } \\
\end{array}$} & \multicolumn{6}{|c|}{ Zone of inhibition (mm) of action of the compounds } \\
\hline & & $\begin{array}{l}6 \mathrm{~b} \\
\mathrm{C}_{6} \mathrm{H} \\
{ }_{13}\end{array}$ & $\begin{array}{l}7 \mathrm{~b} \\
\mathrm{C}_{8} \mathrm{H} \\
17\end{array}$ & $\begin{array}{l}8 \mathrm{bb} \\
\mathrm{C}_{10} \\
\mathrm{H}_{21}\end{array}$ & $\begin{array}{l}9 \mathrm{~b} \\
\mathrm{C}_{12} \\
\mathrm{H}_{25}\end{array}$ & $\begin{array}{l}10 \mathrm{~b} \\
\mathrm{C}_{14} \\
\mathrm{H}_{29}\end{array}$ & $\begin{array}{l}11 \mathrm{~b} \\
\mathrm{C}_{16} \\
\mathrm{H}_{33}\end{array}$ \\
\hline Shigella sonnei. & 400 & -- & 18ロ & -- & -- & -- & 16 \\
\hline Bacillus cereus. & 400 & 10 & -- & 8 & 8 & -- & -- \\
\hline Salmonella typhae & 400 & 5 & 8 & - & - & - & 6 \\
\hline Shigella dyscenteriae. & 400 & -- & 6 & - & -- & -- & 10 \\
\hline $\begin{array}{l}\text { Salmonella paratyphae } \\
A\end{array}$ & 400 & $10 \square$ & 5 & 15 & -- & -- & -- \\
\hline Bacillus subtilis. & 400 & 10 & 10 & 10 & -- & -- & -- \\
\hline Staphylococcus aureus & 400 & 6 & 6 & 9 & -- & -- & -- \\
\hline Escherichia coli & 400 & 6 & 6 & -- & -- & -- & -- \\
\hline Shigella flexneri & 400 & 16 & $\begin{array}{l}12 \\
\square\end{array}$ & $\begin{array}{l}15 \\
\square\end{array}$ & -- & -- & 10 \\
\hline Bacillus megaterium & 400 & -- & -- & -- & -- & -- & -- \\
\hline
\end{tabular}

$6 \mathrm{~b}=3,5$-di-n-hexyloxy benzoyl hydrazine, $7 \mathrm{~b}=3,5$-di-n-octyloxy benzoyl hydrazine, $\quad 8 \mathrm{~b}=3,5$-di-ndecyloxy benzoyl hydrazine, $9 \mathrm{~b}=3$, -di-n-dodecyloxy benzoyl hydrazine $10 \mathrm{~b}=3,5$-di-n-tetradecyloxy

Table-3: Anti-bacterial activity of $(3,4)$ substituted in hydrazine: $\left(\mathrm{R}-\mathrm{C}_{6} \mathrm{H}_{13}-\mathrm{C}_{16} \mathrm{H}_{33}\right.$, Scheme-4)

\begin{tabular}{|c|c|c|c|c|c|c|c|}
\hline \multirow{2}{*}{ Test Organism } & \multirow{2}{*}{$\begin{array}{l}\text { Con of } \\
\text { test } \\
\text { material } \\
\mu \text { gdisk }\end{array}$} & \multicolumn{6}{|c|}{ Zone of inhibition ( $\mathrm{mm}$ ) of action of the compounds } \\
\hline & & $\begin{array}{l}12 \mathrm{~b} \\
\mathrm{C}_{6} \mathrm{H} \\
13\end{array}$ & $\begin{array}{l}13 \mathrm{~b} \\
\mathrm{C}_{8} \mathrm{H} \\
17\end{array}$ & $\begin{array}{l}14 \mathrm{~b} \\
\mathrm{C}_{10} \\
\mathrm{H}_{21}\end{array}$ & $\begin{array}{l}15 \mathrm{~b} \\
\mathrm{C}_{12} \\
\mathrm{H}_{25}\end{array}$ & $\begin{array}{l}16 \mathrm{~b} \\
\mathrm{C}_{14} \\
\mathrm{H}_{29}\end{array}$ & $\begin{array}{l}17 \mathrm{~b} \\
\mathrm{C}_{16} \\
\mathrm{H}_{33}\end{array}$ \\
\hline Shigella sonnei. & 400 & 5 & -- & -- & -- & -- & -- \\
\hline Bacillus cereus. & 400 & -- & -- & 8 & -- & -- & -- \\
\hline Salmonella typhae & 400 & 5 & 6 & 6 & 5 & -- & -- \\
\hline Shigella dyscenteriae. & 400 & 10 & -- & -- & -- & -- & -- \\
\hline $\begin{array}{l}\text { Salmonella paratyphae } \\
\text { A }\end{array}$ & 400 & $10 \square$ & $12 \square$ & 10 & -- & -- & -- \\
\hline Bacillus subtilis. & 400 & -- & -- & -- & -- & -- & -- \\
\hline Staphylococcus aureus & 400 & 8 & 6 & -- & -- & -- & -- \\
\hline Escherichia coli & 400 & -- & 6 & 5 & -. & -- & -- \\
\hline Shigella flexneri & 400 & 16 & 14ㅁ & 6 & -- & -- & -- \\
\hline Bacillus megaterium & 400 & -- & -- & -- & -- & -- & -- \\
\hline
\end{tabular}

$12 \mathrm{~b}=3,4-d i-n-h e x y l o x y$ benzoyl hydrazine, $13 \mathrm{~b}=3,4$-di-n-octyloxy benzoyl hydrazine, $\quad 14 \mathrm{~b}=3,4-\mathrm{di}-\mathrm{n}$ decyloxy benzoyl hydrazine, $15 \mathrm{~b}=3,4$-di-n-dodecyloxy benzoyl hydrazine $16 \mathrm{~b}=3,4$-di-n-tetradecyloxy decyloxy benzoyl hydrazine, $15 \mathrm{~b}=3,4-$ di-n-dodecyloxy benzoyl
benzoyl hydrazine $17 \mathrm{~b}=3,4$-di-n-hexadecyloxy benzoyl hydrazine

Table-4: Anti-bacterial activity of (3, 4, 5) substituted hydrazine: (R- $\mathrm{C}_{6} \mathrm{H}_{13}-\mathrm{C}_{16} \mathrm{H}_{33}$, Scheme-5)

\begin{tabular}{|c|c|c|c|c|c|c|c|}
\hline \multirow{2}{*}{ Test Organism } & \multirow{2}{*}{$\begin{array}{l}\text { Con of } \\
\text { test } \\
\text { material } \\
\mu g \text { disk }\end{array}$} & \multicolumn{6}{|c|}{ Zone of inhibition (mm) of action of the compounds } \\
\hline & & $\begin{array}{l}18 \mathrm{~b} \\
\mathrm{C}_{6} \mathrm{H} \\
13\end{array}$ & $\begin{array}{l}19 \mathrm{~b} \\
\mathrm{C}_{8} \mathrm{H} \\
17\end{array}$ & $\begin{array}{l}20 \mathrm{~b} \\
\mathrm{C}_{10} \\
\mathrm{H}_{21}\end{array}$ & $\begin{array}{l}21 \mathrm{~b} \\
\mathrm{C}_{12} \\
\mathrm{H}_{25}\end{array}$ & $\begin{array}{l}22 \mathrm{~b} \\
\mathrm{C}_{14} \\
\mathrm{H}_{29}\end{array}$ & $\begin{array}{l}23 \mathrm{~b} \\
\mathrm{C}_{16} \\
\mathrm{H}_{33}\end{array}$ \\
\hline Shigella sonnei. & 400 & -- & -- & -- & 5 & - & -- \\
\hline Bacillus cereus. & 400 & -- & -- & 8 & -- & -- & -- \\
\hline Salmonella typhae & 400 & -- & -- & -- & -- & -- & 6 \\
\hline Shigella dyscenteriae. & 400 & -- & -- & -- & -- & -- & -- \\
\hline $\begin{array}{l}\text { Salmonella paratyphae } \\
A\end{array}$ & 400 & -- & -- & 8 & -- & -- & 10 \\
\hline Bacillus subtilis. & 400 & -- & -- & 10 & -- & -- & -- \\
\hline Staphylococcus aureus & 400 & -- & -- & 6 & -- & -- & -- \\
\hline Escherichia coli & 400 & -- & -- & -- & -- & -- & -- \\
\hline Shigella flexneri & 400 & -- & -- & 11 & -- & -- & 10 \\
\hline Bacillus megaterium & 400 & -- & -- & -. & -- & -- & -- \\
\hline
\end{tabular}

$18 \mathrm{~b}=3,4,5$-tri-n-hexyloxy benzoyl hydrazine, $19 \mathrm{~b}=3,4,5$-tri-n-octyloxy benzoyl hydrazine, $20 \mathrm{~b}=3,4,5$ tri-n- decyloxy benzoyl hydrazine, $21 \mathrm{~b}=3,4,5$-tri-n-dodecyloxy benzoyl hydrazine, $22 \mathrm{~b}=3,4,5$-tri-ntetradecyloxy benzoyl hydrazine, $23 \mathrm{~b}=3,4,5$-tri-n-hexadecyloxy benzoyl hydrazine 
Antibiotics used for this experiment:

\begin{tabular}{|c|c|c|c|c|c|c|c|}
\hline №. & Name of antibiotic & 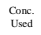 & symbol & No. & Nane of antibiotic & $\begin{array}{c}\substack{\text { Conc. } \\
\text { Ussed }} \\
\text {. }\end{array}$ & Symbol \\
\hline 1 & Cephalothin & 30ug & KF & 7 & Eyythromycin & 15\%g & E \\
\hline 2 & Trimethoprim & ${ }_{\text {sug }}$ & w & 8 & Penicillin G & 10 unis & $\mathrm{P}$ \\
\hline 3 & Gentamycin & 1200 & $\mathrm{CN}$ & 9 & Chloramphneniol & 30ug & c \\
\hline 4 & Strepponycin & 19org & $\mathrm{s}$ & 10 & Metrnidaraole & Shorg & PTZ \\
\hline 5 & Oxyetraxyctine & 30ug & от & 11 & Anpicillin & 254g & AMP \\
\hline 6 & Ammyxcilin & 10urg & AML. & & & & \\
\hline
\end{tabular}

Table-5: Inhibition activities of antibiotics

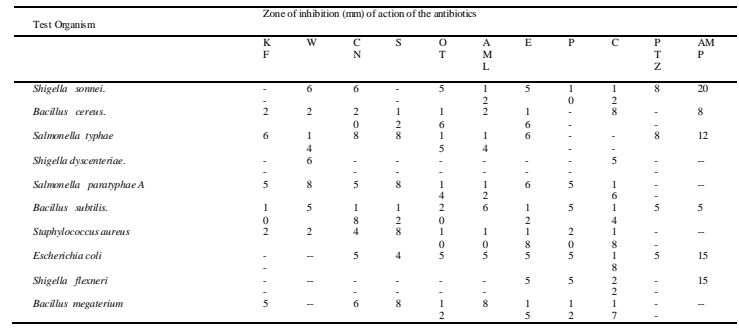

\section{DISCUSSION}

The antibacterial activities of some mixed ligand transition metal complexes of pt (II), $\mathrm{Au}$ (II), Ni (II), $\mathrm{Cu}$ (II) had already been studied ${ }^{[10]}$. A survey of the antibacterial activities of some prepared zolidine derivatives ${ }^{[11]}$ has also been performed. From the results of this paper it is important to point out that out of eleven six antibiotics do not show any significant activity, two inactive against Staphylococcus aureus (inhibition zone values 2$10 \mathrm{~mm})$ table-5. The values are much more less to damage the bacterial cell of the bacteria. It is noted that 4-n-hexyloxy benzoyl hydrazine shows highest activity against Staphylococcus aureus (inhibition zone value $16 \mathrm{~mm}$ ) and has highest activity against Bacillus cereus (22mm).Therefore 4-n-hexyloxy benzoyl hydrazine may be used as a good antibacterial agent against Staphylococcus aureus but further trial is necessary. 4-n-decyloxy benzoyl hydrazine has highest activity against Salmonella typhae and has moderate activity against Shigella dyscenteriae, Salmonella paratyphae A, Bacillus subtilis and Staphylococcus aureus. 4-n-nonyloxy benzoyl hydrazine do not have any activity against Shigella sonnei, Bacillus cereus, Salmonella typhae, Shigella dyscenteriae, Salmonella paratyphae A, Bacillus subtilis, Shigella flexneri and less sensitive against Staphylococcus aureus, Escherichia coli and Bacillus megaterium.3,5substituted hydrazines are more active, some 3,4substituted hydrazines are moderately sensitive and some 3,4-subtituted hydrazines do not have any activity and are less sensitive, the activity series is $3,5>3,4>3,4,5$ hydrazine. 3,5-di-nhexadecyloxybenzoylhydrazine shows highest activity against Shigella sonnei $(16 \mathrm{~mm})$. 3,5-di-noctyloxybenzoylhydrazine shows highest activity against Shigella sonnei (18mm) for twelve hours. 3,4-di-n-hexyloxybenzoylhydrazine shows highest activity against Shigella flexneri $(16 \mathrm{~mm})$.

\section{CONCLUSION}

Single chain hydrazines are more active than double and triple chain hydrazines. Anti-microbial activity decreases as the number of carbon of single and double chain hydrazine increases, $\left(\mathrm{C}_{6}>\mathrm{C}_{7}>\mathrm{C}_{8}>\right.$ $\mathrm{C}_{9}>\mathrm{C}_{10}$ single and double chain hydrazine.) Double chain hydrazines $(3,5$ or 3,4$)$ are more active than triple chain hydrazines, $(3,5>3,4>3,4,5$ hydrazine). The poor activity of other hydrazines (3, 4,5 , some 3,5 , some 3,4 , and single chain) may be due to the hydrophobic properties of these hydrazines which may inhibits permeation through the lipoid layers of microorganism membranes. The significant activity of hexyloxybenzoyl hydrazine and heptyloxybenzoyl hydrazine against gram positive and gram negative bacteria may be (formation of inhibition zone 8 to $22 \mathrm{~mm}$ with most of the test bacteria) due to their liophilicity of the bacterial cell membrane.

\section{ACKNOWLEDGEMENTS}

The authors are grateful to the Director General of Bangladesh Livestock Research Institute Saver Dhaka, for the permission of this research work in this institution and also grateful to the head of the department of animal health research division for their cordial cooperation to use well equipped bacteriology laboratory and also grateful to Food and Nutrition Department of Dhaka University for pure culture organism.

\section{REFERENCES}

1. Treshchaeina EM, Konovalova AL, Presnov MA, Chapurina LF and Belichuk NI(1979). Antitumour properties of mixed coordination compounds of copper (II) and $\alpha$-Aminoacids. Dokl. Akad. Nauk.248, 1273-1276.

2. Doadrio AD, Craciunescu B, Sosa and Fruma A (1979). Relation between the Structures and antitumour activity of complexes palatinate and cuprate salts, An. R.Acad. Farm.45, 497.

3. Heinish L, Fleck WF and Jacob HE (1980). Copper (II) complexes with N-heterocyclic formylisothiosemicarbazone with antibacterial and $\beta$-lactamase inhibiting effect. Z. Allg. Microbiol. 20, 679.

4. Baranyi A and Feher O (1979). Convulsive effects of 3-aminopyridine on cortical neurons Electroencephalogr. Clinical Neurophysio., 47, 745.

5. Szente M, Feher O and Gyuris T (1984). The effects of aminopyridines on the critical evoked potentials. Acta Physiol. Hung. 63,197.

6. Sultana CM, Rahman AA, Bari, Banu MLA, Islam MS, Khatun NA and Sadik G (2003). In 
vitro antimicrobial screening of three cadmium complexes and two additional compounds of antimony and arsenic. Pakistan Journal of Biological Sciences. 6, 525-527.

7. Islam MS, Farooque MA, Bodruddoza MAK, Mosaddik MA and Alam MS (2002), Antimicrobial and toxilogycal studies of mixed ligand transition metal complexes of Schiff bases. Online Journal of biological Science. 2, 797-799.

8. Hargital C, Egyhaze K and Markus G (1992). Trends of changes in the antibiotic sensitivity of under pathogen. Magyar Allatorvosok Lapija. 47(8):429-432.

9. Sarma DK (2002). A Text Book of Veterinary Bacteriology and Bacterial Diseases.Kalyani Publishers, X-press graphics, New Delhi-11002.

10. Akbor MA, Ahsan MA, Akter S, Islam MS, Ahter N and.Hossain MM (2009). The antibacterial activities of some mixed ligand transition metal complexes of some dibasic acids with amine bases. Jahangirnagar Univ. J. Sci. 32(1), 1-6.

11. Haque MZ, Ali MU (2007). Synthesis and biological activities of some new zolidine derivatives from 1-substituted-3-N-aleuritamido thiocarbamides, Journal of the Bangladesh Chemical Society. 20(1), 99-103.

12. Carcelli M, Mazza P, Pelizzi C, Pelizzi G, and Zani F (1995). Antimicrobial and Genotoxic activity of 2, 6-Diacetylpyridine Bis ( Acylhydrazones) and Their Complexes with Some First Transition Series Metal Ions. X-ray Crystal Structure of a Dinuclear Copper (II) Complexes. Journal of Inorganic Biochemistry.57,43-62. 